\title{
Inventer et innover quand personne ne veut payer : Le nettoyage des débris spatiaux
}

\author{
Inventing and innovating when no one wants to pay:
} Cleaning up space debris

\author{
Emilie Hertig ${ }^{1}$, Lucile Van Box Som ${ }^{2}$ et Dominique Vinck ${ }^{3}$ \\ ${ }^{1}$ École Polytechnique Fédérale de Lausanne, Suisse, emilie.hertig@epfl.ch \\ ${ }^{2}$ Observatoire de Paris, France, lucile.vanboxsom@gmail.com \\ ${ }^{3}$ STSLab, Institut des Sciences Sociales, Université de Lausanne, Suisse, dominique.vinck@unil.ch
}

RÉSUMÉ. L'incitation marchande à investir dans l'invention de solutions et leur adoption serait souvent le moteur de l'innovation, mais lorsqu'il s'agit d'investir pour le bien commun, elle ne joue pas. C'est le cas en matière d'élimination des déchets spatiaux. La biographie d'une innovation dans ce domaine documente la culture d'ingénierie qui accompagne l'invention d'une solution qui doit aussi prendre en compte une problématique sociétale (la tragédie des communs) : comment innover lorsque personne n'est prêt à payer pour l'adoption de l'invention ? Avec l'étude biographique du projet Clean Space, l'article montre que, loin de se réduire à l'affaire de quelques héros, l'innovation mêle des collectifs qui explorent et entrelacent des questions de techniques, de bien commun, de financement, de normes internationales, de pédagogie et des rêves. Cette biographie d'innovation débute avec un projet pédagogique de microsatellite, confronté à des retournements de situation sources d'apprentissages, qui enrichit la culture technique du collectif concerné.

ABSTRACT. The market incentive to invest in the invention and adoption of solutions would often be the driving force behind innovation, but when it comes to investing for the common good, it does not apply. This is the case with innovation in space waste disposal. The story of innovation in this field documents the engineering culture that accompanies the invention of a solution, which must also take into account a societal problem (the tragedy of the commons): how can we innovate when no one is willing to pay for the adoption of the invention? Using the Clean Space project as the focus, the article demonstrates that - far from being reduced to the business of a few heroes - innovation mixes different collectives that explore and interweave technical issues, the common good, financing, international standards, pedagogy and dreams. This paper begins with a teaching project based around a microsatellite; the result is a reversal of learning situations which enriches the technical culture of the collective concerned.

MOTS-CLÉS. Invention, innovation, culture d'ingénierie, bien commun, tragédie des communs, espace, microsatellite. KEYWORDS. Invention, innovation, engineering culture, common good, tragedy of the commons, space, microsatellite.

Reconstituer la biographie d'une innovation est un bon moyen pour rendre compte de la complexité de l'assemblage hétérogène que réalisent les acteurs [LAT 05] pour qu'une nouveauté (technique) prenne place en société. Loin de se réduire à la concrétisation d'une idée géniale ou à la suite logique de développements technologiques, l'innovation est le fruit de processus historiques et situés. L'analyse de sa biographie met en évidence l'influence des conditions de son surgissement, les besoins auxquels ses acteurs tentent d'apporter une réponse, ainsi que les ressources et contraintes de son développement, de sa socialisation et de son inscription dans la société. L'histoire des techniques [EDG 98] et la sociologie de l'innovation [AKR 91] ont contribué à le démontrer. Revenir sur l'innovation en partant de cas concrets est toutefois toujours source d'enseignements et contribue au développement d'une culture technique.

\section{Approche conceptuelle et méthode}

La culture technique est l'ensemble de connaissances, valeurs, manières d'être et de faire, ressources langagières, normes et convenances, habitudes et habiletés, et objets qui permettent à un collectif, techniques comprises, de faire société. Elle constitue une manière de penser et de relier les techniques à d'autres éléments du monde et de les intégrer en un ensemble porteur de sens. Elle permet à une 
société d'explorer et d'évaluer l'intégration de nouveautés techniques, de les socialiser et de les naturaliser (ou trivialiser). La culture technique ne se réduit donc pas aux connaissances et savoir-faire qui permettent d'agencer les moyens, matériels et opératoires, permettant d'assumer une fonctionnalité. Elle comprend aussi les manières d'être et de faire permettant de tirer profit des techniques dans des environnements variés et de faire évoluer les agencements associés en fonction de questions de sens, de valeurs et des visées collectives. Elle concerne nos rapports aux techniques en société et les mondes que construits avec elles [CHO 17b]. Elle implique de les penser autrement que comme agencements de ressources matérielles en un schème opératoire efficient car tous ses éléments sont aussi façonnés par des dynamiques collectives. Les techniques portent en elles la mémoire de la société et des savoirs mobilisés [JAC 17]. Elles sont un bout de société [LAT 91] et un moyen de poursuivre la politique par d'autres moyens [LAT 88].

Correspondant à des domaines ou métiers particuliers où des cultures techniques spécifiques sont formées de savoirs et savoir-faire formels et tacites, de réalisations exemplaires, de pratiques, de technolectes [GAR 17], de règles de conception et de nombreux artefacts. Bien que portant sur des objets et savoir-faire opératoires, elles portent toujours aussi sur les relations entre personnes, l'organisation du travail et la conduite des affaires [VIN 14]. Imprégnées de valeurs (rigueur, rationalité, progrès...) et de sens, elles portent des visions du monde souvent naturalisées et tacites. Ces cultures techniques sont restreintes à un domaine d'activité, côté conception et développement plutôt qu'usage en société, maintenance et réparation. De ce fait, elles alimentent des représentations d'objets techniques sans culture [DID 17] au niveau de la formation académique, ce qui est moins le cas pour les gens de métier qui ont l'expérience du «terrain», se confrontent à d'autres métiers, à des utilisateurs, à des retours d'expérience. Le travail des ingénieur·e $\cdot s$ consiste ainsi souvent à trouver des solutions viables en les négociant et en coordinant des flux d'information et d'acteurs [WIL 14]. Dans le cas traité dans cet article, elles concernent les spécialités de jeunes ingénieur·e.s venant notamment des télécommunications, de la robotique, de microtechnique et de l'électronique.

Des cultures polytechniques, développées dans la formation ou par l'expérience, juxtaposent ou intègrent différentes cultures techniques. Imprégnées de savoirs, méthodes et savoir-faire systématisés et formalisés, dans le cadre de la formation ou de sociétés professionnelles, ainsi que des manières de penser et de se comporter, elles véhiculent aussi des savoirs et méthodes du management, de l'organisation du travail et de la conduite des affaires. Elles véhiculent une vision distante de la technique - réduite à des principes de fonctionnement et à des mécanismes, sans expérience sensible -, de sa genèse et de son devenir (maintenance, usages et fin de vie), ce qui alimente une représentation de la technique hors sol et hors société. Elles sont toutefois aussi imprégnées de visions du monde et de politiques qui parfois s'affrontent (e.g. concernant les architectures informatiques).

Nous parlerons de cultures d'ingénierie à propos des pratiques, savoir-faire, connaissances et valeurs à l'œuvre dans l'ingénierie et qui portent sur l'intégration de ressources hétérogènes, l'hybridation de savoirs à visée fonctionnelle, associées à des finalités, valeurs et visions du monde. Elles concernent des compétences d'assemblage de cultures techniques spécifiques, orientées un souci de résolution de problèmes (dont les grands défis planétaires et de société), de pragmatisme et de rationalité qui fait feu de tout bois. Elles traduisent parfois une sensibilité à des valeurs comme l'équité, la durabilité, la responsabilité vis-à-vis des générations futures et le sentiment d'être acteur politique. Elles sont imprégnée de connaissances portant sur la société. Dans le cas qui nous occupe, la culture d'ingénierie est marquée par une culture du spatial avec son univers de contraintes et de manières de faire.

Enfin, par culture de l'innovation, nous nous référons à l'ensemble de savoirs, savoir-faire, valeurs et pratiques lié à l'idée de nouveauté et à son introduction, Cette culture se réduit souvent à des prescriptions managériales devant permettre de créer les conditions favorables à l'invention et à son inscription dans la société (souvent réduite à un marché), peu sensibles à la matérialité et aux questions du sens et de valeurs autre qu'économiques, et de vision pour la société. Cette culture d'innovation, qu'on trouve dans les manuels et les discours, n'apporte pas de solution spécifique aux grands défis 
contemporains tant sa culture technique est pauvre [CHO 17a]; elle s'apparente à une idéologie [GOD 17] du progrès et des mérites de la compétition, plus qu'à une culture technique. Dans le domaine du développement durable et de la transition, des cultures de l'innovation soucieuces de changements sociétaux et planétaires se développent comme ce fut le cas avec une vision des nanotechnologies, dans les années 1980, qui offraient du rêve à qui se souciait de l'avenir de la planète (idée qu'il faudrait un jour réduire la quantité de matière et d'énergie consommée) (selon C. Joachim [MAN 08]).

En prenant le cas d'une invention qui relève de la recherche technologique dans le domaine spatial mobilisant surtout des établissements d'enseignements, l'article se situe aux marges du paradigme dominant de l'innovation contemporaine, focalisée sur la production industrielle de biens de consommation. En outre, le cas étudié, concernant un développement en cours, a le mérite d'être une action en situation d'incertitudes [CHA 09]. Son analyse biographique rend compte de la genèse de l'invention et du cheminement en vue de son adoption, sa mise en œuvre, sa diffusion et son utilisation effective. L'innovation est ici conçue comme une invention sanctionnée positivement par des acteurs qui l'adoptent et consentent des investissements (humains et financiers) afin d'en tirer bénéfice (économique, politique, symbolique ou pour le bien-être de la planète ou de la société). Un fossé sépare l'invention de l'innovation.

L'examen biographique de l'invention devrait nourrir la culture technique en y instillant une réflexion portant sur les conditions et processus du développement technologique effectif et sur les incertitudes, y compris sociétales, à prendre en compte. L'article rend compte de l'évolution incrémentale d'une culture d'ingénierie et montre que le succès de l'innovation dépend de la prise en compte de problématiques initialement peu présentes, en l'occurrence la question du devenir d'inventions en l'absence d'incitation économique. La biographie de l'innovation rend alors compte d'un processus d'apprentissage collectif en prise avec des questions comme: qu'advient-il des inventions lorsque personne n'est prêt à payer pour leur adoption ? Quels mécanismes conduisent à l'innovation dans ce cas? Classiquement, l'innovation s'explique notamment par l'intérêt que des acteurs ont à investir dans l'invention de solutions, puis dans leur adoption. Cet intérêt est pécuniaire si la nouveauté permet d'économiser des ressources ou de générer des revenus. Il peut ne pas être pécuniaire si l'adoption s'explique par l'espoir d'une satisfaction (e.g. meilleure qualité de vie, accomplissement d'un rêve), par une finalité valorisée (e.g. la survie de la planète) ou par une pression sociale (e.g. réduction des émissions de $\mathrm{CO}_{2}$ ). Pour les acteurs industriels, l'intérêt est souvent financier. Cependant, lorsqu'il s'agit d'investir pour le bien commun (e.g. assurer la propreté de l'environnement) et au bénéfice d'autrui, rien ne garantit que l'investissement soit également rentable pour soi-même. Tel est le cas de l'innovation en matière d'élimination des déchets spatiaux.

La présente biographie d'innovation contribue à la connaissance des modes d'existence des innovations en mettant en lumière son épaisseur sociétale. Elle nourrit la culture technique en contribuant à la constitution d'un patrimoine commun. L'approche biographique implique de prendre en compte les contextes pertinents, dont des cultures techniques spécfiques, des besoins et défis, des réseaux d'acteurs, des cours d'actions qui prennent la forme d'explorations, de créations, de transferts de connaissances, de combinaisons entre domaines, de contournement, d'adaptation à l'imprévu, etc. Notre approche, héritée de la sociologie de l'innovation [VIN 18], est sensible aux modalités d'existence des objets et aux dynamiques de leur genèse, circulation, usage et maintenance.

Cette biographie repose sur des matériaux réunis entre 2013 et 2019 et comprend la collecte de documents disposnibles sur les sites web des acteurs concernés, de reportages télévisés et d'entretiens réalisés par des étudiant $\cdot \mathrm{e} \cdot \mathrm{s}$ ingénieur.e.s. ${ }^{1}$

\footnotetext{
${ }^{1}$ L. Van Box Som et L. Germeau, dans le cadre du cours de Master "STS - Dynamique de I'innovation" en 2013-2014. Travail repris et approfondi par E. Hertig et D. Vinck en 2017-2019.
} 


\section{Biographie d'une innovation}

\subsection{Culture d'ingénierie et innovation pédagogique}

La genèse du projet Clean Space One se situe en 2009 lors du lancement de SwissCube ${ }^{2}$, premier satellite entièrement construit en Suisse. Développé par l'École polytechnique fédérale de Lausanne (EPFL) en partenariat avec l'Université de Neuchâtel et cinq écoles d'ingénieur(e/s) suisses [NOK 09] comme projet pédagogique permetant à des étudiant·es $\mathrm{s}$ d'étoffer leur culture technique en acquérant de l'expérience sur le cycle complet de développement d'un satellite. Le projet vise aussi à développer leur culture d'ingénierie en termes d'autonomie, de flexibilité, de communication et d'intégration des savoirs au sein d'une équipe composée d'une quinzaine de laboratoires aux cultures techniques spécifiques. Plus de 250 étudiant·e·s, accompagné·e·s de chercheur-euse·s relevant de différentes spécialités en microtechnique, sciences des matériaux, télécommunications, physique et électronique interviennent au cours du temps. Le milieu porteur peut ainsi être caractérisé par sa culture académique d'ingénierie, soucieuse à la fois de s'attaquer à des défis technologiques - qui, si possible, font sens pour la société -, d'en tirer de la connaissance publiable dans de bons supports scientifiques et de

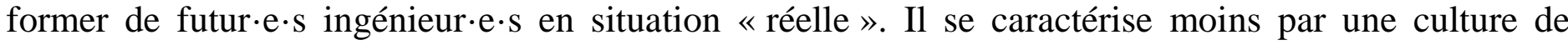
l'innovation que par une culture d'ingénierie. Le projet est dirigé par la professeure Muriel Richard, ingénieure au Swiss Space Center de 1'EPFL depuis 2005. Diplomée de 1'Ecole Polytechnique Féminine dans le domaine de l'ingénierie aéronautique et aérospatiale et du California Institute of Technology (USA) en génie mécanique, elle a travaillé au Jet Propulsion Laboratory (JPL) de la NASA, pour le Programme Mars, pour l'Advanced Propulsion Technology Group avant de devenir ingénieure principale du Deep Space Mission Architecture Group du JPL. Elle apporte au projet Swiss Cube et dans la formation des étudiant·e·s son expérience et une culture d'ingénierie spatiale, en termes de conception de satellites pour l'exploration spatiale, commune aux acteurs du domaine, qu'ils relèvent de la recherche, de l'ingénierie, de l'administration et du management des projets spatiaux, y compris la communication médiatique et un public d'amateurs éclairés.

L'objectif scientifique de SwissCube est de mesurer l'intensité de l'airglow, couche lumineuse de la haute atmosphère. Dans ce but, l'équipe lausannoise conçoit un télescope spécial et un concept de capteur terrestre à petit prix. Ce projet, réalisé en trois ans, effectue son lancement le 23 septembre 2009 depuis le pas de tir de Satish Dawan, en Inde, avec trois autres petits satellites. La durée de vie de Swiss Cube est de quatre mois, mais, en 2019, il est toujours en orbite, malgré le bombardement des particules cosmiques sur ses composants électroniques et, depuis 2016, des signes de fatigue ; il est classé au $4^{\text {ème }}$ rang de la longévité des picoosatellites. ${ }^{3}$ Il fournit chaque jour des télémétries, démontrant ainsi sa réussite.

SwissCube appartient à la catégorie des CubeSats, satellites cubiques de $10 \mathrm{~cm}$ de côté, d'un Watt de puissance et d'environ un kilogramme, selon un concept élaboré et diffusé par des chercheurs de l'Université polytechnique de Californie et de l'Université de Stanford pour permettre aux universités et industriels de lancer des expériences scientifiques à coût réduit. Ces picosatellites sont devenus un moyen privilégié pour tester de nouveaux concepts et systèmes embarqués. Le projet SwissCube prouve ainsi les compétences de l'EPFL, notamment en testant du matériel non certifié pour le spatial, fabriqué avec des composants low cost, peu utilisés dans l'espace, et en miniaturisant toutes les fonctions d'un grand satellite sur un petit. Il contribue au développement d'une culture en ingénierie spatiale et sa reconnaissance internationale, et consolide la participation de la Suisse dans l'Agence spatiale européenne (ESA) en tant qu'état membre ayant démontré ses compétences techniques. L'ESA soutient ce projet en autorisant l'équipe lausannoise à utiliser sa station sol de Darmstadt, l'ESOC (Centre Européen des Opérations Spatiales) pour communiquer avec SwissCube. 


\subsection{Retournement dramatique : le surgissement d'une problématique sociétale}

Cependant, à quelques jours du lancement de SwissCube, l'ESOC retire son soutien logistique au projet Swiss Cube. La raison avancée pour justifier ce retournement est le non-respect de la ligne de conduite de l'ESA concernant la fin de vie des satellites en orbite [ESA 06]. Le satellite à la fin de sa vie opérationnelle doit sortir de son orbite pour se désintégrer dans l'atmosphère, atteindre une orbite cimetière ou être recyclé pour une autre mission. L'objectif est de diminuer la production de déchets spatiaux en orbite autour de notre planète.

Depuis la première mise d'un satellite en orbite en 1957, le nombre de déchets spatiaux n'a cessé d'augmenter, au point d'être devenu un problème pour les missions spatiales. Il y a environ vingt-neuf mille [ESA 18] débris spatiaux de plus de dix centimètres de diamètre qui orbitent à des vitesses pouvant dépasser $28^{\prime} 000 \mathrm{~km} / \mathrm{h}$. En cas de collision, ces débris peuvent gravement endommager voire causer la perte d'un satellite. ${ }^{4}$ La première collision importante, qui permit de prendre conscience du problème, a eu lieu en 2009 entre le satellite américain opérationnel Iridium 33 et le satellite soviétique épave Cosmos 2251. En 2013, moins d'un mois après son lancement, le premier satellite équatorien est entré en collision avec le réservoir d'un lanceur russe lancé en 1985, entrant ensuite en rotation incontrôlée empêchant alors toute communication avec le sol. La menace à terme tient à l'augmentation du nombre de débris spatiaux car chaque impact génère un nombre important de fragments de petite taille qui peuvent, à leur tour, causer des accidents et produire de nouveaux débris. Une telle réaction en chaîne (syndrome de Kessler) pourrait provoquer une augmentation exponentielle du nombre de débris qui rendrait l'orbite basse inaccessible [HAL 14]. Ce phénomène constitue la réelle menace. Pourtant, elle est souvent soit ignorée soit minimisée par manque d'intérêt financier.

\subsection{Culture d'ingénierie : concevoir une solution technique}

La fin de vie de SwissCube n'a été ni pensée ni conçue lors du développement du satellite, lequel fut alors montré du doigt par la communauté spatiale comme exemple de satellite voué à devenir un déchet spatial. SwissCube est néanmoins lancé, sans que la problématique de sa fin de vie n'ait trouvé de solution. Malgré la frustration due au retrait de l'ESOC, SwissCube représente un succès pour l'équipe du projet ; deux autres stations au sol sont utilisées pour communiquer avec lui, l'une à l'EPFL, l'autre à HE-Fribourg.

La problématique des déchets spatiaux devenant importante pour la communauté européenne spatiale, l'équipe du projet SwissCube entend alors trouver une solution pour la fin de vie des petits satellites et prouver que SwissCube ne deviendra pas un déchet spatial. Elle lance le programme Clean$m E$ en 2009 qui rassemble différents laboratoires de l'EPFL (dont des laboratoires de robotique) dont l'objectif est de concevoir et développer une technologie permettant d'aller chercher des débris en orbite et de les ramener dans l'atmosphère terrestre pour qu'ils s'y désintègrent. Comment aller chercher des débris ? Avec quelles technologies ? Quelles sont les exigences pour une telle mission ? Quel type de débris doit-on viser ? Les équipes du programme conduisent alors différentes études pour préciser les objectifs et débouchent sur un premier rapport, en 2010, définissant l'objectif du programme Clean- $m E$, à savoir aller chercher en orbite cinq à dix débris de taille entre $10 \times 10 \times 10 \mathrm{~cm}^{3}$ et $15 \times 15 \times 15 \mathrm{~cm}^{3}$ [SWI 10]. Un premier projet de satellite chargé de désorbiter un débris, appelé CleanSpace One, est proposé et engagé. Le but à long terme est d'ouvrir la possibilité de concevoir et construire, à partir des technologies développées, une famille de satellites destinée au nettoyage des orbites proches. Il s'agit de proposer et de commercialiser une famille de systèmes clés-en-main et adaptables à plusieurs types de satellites à désorbiter, en taille et en masse.

\footnotetext{
4 https://www.futura-sciences.com/sciences/actualites/astronautique-iss-nouvelle-fois-sous-menace-debris-spatial-36048/ en 2012; https://www.lexpress.fr/actualite/sciences/les-debris-spatiaux-une-pollution-invisible-mais-dangereuse 1741408.html en 2015; https://www.lexpress.fr/actualite/sciences/debris-spatiaux-un-grand-nettoyage-s-impose_1977156.html, consulté le 15 avril 2019.
} 
L'idée est de mettre au point des solutions afin que chacun s'occupe de ses propres débris en orbite. Bien que les débris les plus dangereux soient les plus gros, le projet entend tester des technologies pouvant être utilisées pour effectuer un retrait actif de débris (Active Debris Removal - ADR) dans le cas d'un microsatellite. Le but de Clean Space One est de démontrer la faisabilité d'un rendez-vous sur orbite, la faisabilité des opérations de capture de satellite [RIC 13] et la fiabilité des instruments d'optique nécessaires au bon déroulement de la mission. A ce stade du projet, plusieurs solutions techniques sont envisagées et l'objectif est d'établir la faisabilité au niveau du hardware. Des études détaillées et la définition des sous-systèmes viendront dans un second temps.

Pour cette démonstration, des ressources sont nécessaires. Parralèlement au projet Clean Space One, l'équipe lausannoise collabore avec le CNES (Centre Nationale d'Etudes Spatiales français) pour tester différentes technologies d'ADR et avec l'ESA sur la recherche en orbite des débris spatiaux. L'idée est de concevoir des technologies utilisables quelle que soit la taille du débris et d'établir une loi d'échelle permettant le transfert de technologie entre différentes missions. Le but de la collaboration est d'acquérir de l'expérience et de mieux comprendre l'ADR.

La biographie de cette innovation débute donc d'une manière relativement classique en partant d'un problème identifié - l'élimination des débris spaciaux -, dans un contexte technologique particulier - le domaine spatial, la prolifération des débris et la multiplication des alertes -, mais aussi institutionnel puisqu'il s'agit d'un second projet pédagogique, à l'occasion duquel se joue la réputation d'une institution de recherche technologique. Le contexte du projet Clean Space One est politiquement singulier puisque l'institution a été montrée du doigt suite au projet SwissCube et son non-respect d'une ligne de conduite qui prend de l'importance au regard de la prégnance des précepts de développement durable.

\subsection{Culture de l'ingénierie spatiale : la construction d'un compromis}

Au début du projet Clean-mE, plusieurs scénarios sont étudiés pour capturer et désorbiter des débris. La sélection des méthodes possibles est guidée par la nécessité de contrôler le désorbitage, tout en gardant à l'esprit que le projet vise des débris de petite taille. La première méthode est l'enlèvement passif ; elle consiste à piéger le débri dans un grand «filet» lancé par un satellite chasseur afin de désorbiter le débri sous l'action de la trainée du filet. Cette solution ne garantit pas un total contrôle du désorbitage mais elle a l'avantage de réduire la masse du satellite chasseur. La seconde solution est le désorbitage du débri en passant par la capture, suivie d'une modification de la vitesse et de la trajectoire du débri, par exemple pour le ramener dans l'atmosphère terrestre où il se consumerait. La masse et la taille des débris sont alors des paramètres essentiels alors qu'ils ne sont pas nécessairement connus d'avance. Un système capable d'agir de façon fiable, alors qu'il se déplace à une vitesse de plusieurs centaines de mètres par seconde par rapport à la cible, est difficile à envisager pour un petit satellite. Enfin, la dernière proposition consiste à donner une vitesse suffisante au satellite chasseur pour que le satellite et le débri capturé pénètrent dans l'atmosphère à un moment déterminé. Bien que cette solution implique d'augmenter la masse du chasseur, elle offre le meilleur contrôle sur l'exécution des différentes étapes de la mission. La masse totale des débris collectés devient alors le facteur limitant dans la construction des satellites chasseurs. Cette dernière solution est adoptée pour le projet Clean-mE. Elle se traduit par un corps de satellite en deux parties : la première contient différents sous-systèmes, dont le système de communication et l'ordinateur de bord; la seconde contient la charge utile, en particulier les mécanismes de capture.

La réussite de la mission dépend de la capacité à concevoir une solution tenant compte de nombreuses contraintes dont la petite taille du satellite et sa masse. Les exigences à prendre en compte changent se discutent au début du projet jusqu'à l'établissement d'un compromis portant sur la taille des débris à attraper; Clean Space One, microsatellite d'environ $30 \mathrm{~kg}$, s'attaquera à des débris d'une taille équivalente à trois unités de CubeSat, soit un volume de trois mille centimètres cube (trois litres). Les exigences sont les suivantes : le système de capture doit être capable de fonctionner même s'il est 
partiellement endommagé et assurer la liaison avec le débris depuis le moment de la capture et au cours de son remorquage jusqu'à la rentrée atmosphérique. Pour répondre à ces exigences, plusieurs configurations sont étudiées, notamment différents types de bras : bras parapluie, bras-poignet et bras crayon de plomb. A ce stade du projet, le bras parapluie est perçu comme ayant de nombreux avantages (simplicité, masse, temps de construction) malgré une manœuvrabilité inférieure à celle du bras-poignet. Pour étudier cette solution, le Swiss Space Center demande l'aide du Laboratoire de microsystèmes pour des technologies spatiales qui mobilise un étudiant pour évaluer un système de pince et faire un état de l'art. La recherche aboutit à l'identification d'une solution peu consommatrice en énergie et en masse tout en étant très flexible. Après une étude et des tests pour Clean Space One, le système est sélectionné et présenté lors d'une conférence internationale en mars 2014.

Les défis techniques (détection et identification du débris cible, approche et rendez-vous en orbite) conduisent les chercheurs à mobiliser un mélange de technologies, certaines étant disponibles, d'autres devant être miniaturisées et adaptées au projet. La première phase du projet débute en septembre 2014 par une analyse fonctionnelle du système et une analyse des spécificités. Elle débouche sur la conception préliminaire du satellite et la mise en évidence de différents compromis. Cette étude de faisabilité formalise les exigences auxquelles le satellite devra répondre. Le projet étant prévu pour se réaliser en quatre années, le lancement se ferait en 2018. Cependant, la consolidation du financement est plus difficile qu'il n'y semblait ${ }^{5}$; le lancement n'a donc pas eu lieu. Une fois les financements rassemblés, il faudrait quatre ans pour réaliser la mission, ce qui la situe en 2021, peut-être 2022, si tout se passe bien. ${ }^{6}$

La biographie de cette innovation est ainsi scandée en plusieurs étapes a priori classiques, débutant par la recherche de concepts, puis de principes de solution, leur évaluation critique, le choix d'une stratégie et la concrétisation de la solution, la réalisation de nombreux tests, avant la réalisation d'un lancement et de la démonstration de la solution.

\section{Pourquoi tant d'incertitudes?}

Les défis de Clean Space One concernent la faisabilité technique, mais aussi son financement et donc sa capacité à convaincre adopteurs et investisseurs. L'incertitude pèse alors sur son avenir et la capacité de cette technologie à se diffuser, ce que l'étude biographique aide à comprendre. Souvent, les innovations échouent parce que leurs conditions d'usage et d'insertion dans des agencements sociotechniques préexistants n'ont pas été prises en compte ou que la nouveauté ne fait pas sens pour ses utilisateurs potentiels. Dans le cas présent, le contexte de la prolifération des déchets et du souci d'un développement durable donnent du sens à l'innovation. Notre hypothèse est plutôt qu'ici l'incertitude vient du fait que le problème de la tragédie des communs n'a pas été anticipé.

\subsection{Un oubli de l'invention : la tragédie des communs}

La poursuite du projet, à savoir le lancement du satellite Clean Space One, celui d'autres démonstrateurs qui lui succéderaient et la diffusion de ce genre de technologie dépend manifestement d'un financement peu évident à assurer. Les porteurs du projet se demandent comment amener les acteurs du spatial à adopter l'invention et à soutenir sa mise en oeuvre. Surgissent alors les questions de motivation et d'incitations financières, juridiques et morales. Qu'est-ce qui peut pousser un industriel ou une nation à investir dans une technologie qui nettoie l'espace ? Leur conscience écologique suffit-elle à le inciter à nettoyer l'espace pour autrui ?

Durant plus de cinquante ans, la conquête de l'espace a pris la forme d'une compétition entre nations qui, pour leur prestige, leur suprématie ou le développement de leurs infrastructures terrestres (télécommunications, navigation, etc.), ont consenti de gros investissements publics et créé des agences

\footnotetext{
${ }^{5}$ https://espace.epfl.ch/CleanSpaceOne 1, dernière consultation le 29 octobre 2018.

6 https://avisdexperts.ch/videos/view/6863, dernière consultation le 16 avril 2019. 
imposantes (NASE, ESA, etc.). Or, depuis le début des années 2000, l'espace a changé. Le new space se peuple désormais de nombreux acteurs émergents. Des pays comme la Chine sont entrés dans la course, mais aussi des entrepreneurs utopiques, dynamiques et richissimes comme Elon Musk de PayPal, Tesla et SpaceX, Jeff Bezos d'Amazon, Larry Page de Google, Richard Branson de Virgin, Robert Richard de Moon Express. L'innovation technologique permet désormais à de petites organisations de faire ce que seules les grandes nations pouvaient accomplir. Des projets de colonisation et d'exploitation l'espace, de Mars, de météorites ou d'Europa, une lune de Jupiter où il y a probablement de l'eau et peut-être de la vie, prennent consistance.

Se pose alors la question d'un nouvel ordre inter-planétaire et d'un droit international. Ce droit va-til rendre responsables les lanceurs de satellites des collisions qu'ils peuvent occasionner? Quelles instances seraient mobilisées pour régler les conflits qui surgiront? Ne s'agit-il pas plutôt de miser sur la conscience morale et sur le souci des acteurs de l'aérospatial de protéger leur réputation en s'occupant de leurs propres déchets ? Le succès d'innovations comme Clean Space One dépend de cette autre trajectoire des satellites dans l'espace du droit international.

Les acteurs de Clean Space One en font toutefois une question technologique. Imprégnés d'une culture technique relevant du spatial, ils intègrent les contraintes et manières de faire du domaine dans la conception d'une solution technique et supposent que les acteurs (publics et privés) concernés par l'élimination des débris ont une motivation évidente pour la gestion durable de l'espace. De fait, la Suisse s'en préoccupe. L'ESA développe des technologies pour traiter les gros débris européens. Les États-Unis, le Japon, la Russie se montrent conscients du problème bien qu'ils soient moins actifs dans ce domaine. ${ }^{7}$ Toutefois, malgré les alertes, la motivation n'est pas au rendez-vous ; Clean Space One en souffre et découvre la tragédie des communs.

La tragédie des communs décrit une compétition pour l'accès à un bien commun, qui est une ressource limitée, menant à la surexploitation de la ressource. Garrett Hardin [HAR 86] considère ainsi que la propriété commune d'une ressource conduit nécessairement à sa ruine, sauf si elle est soit nationalisée, soit privatisée. Elinor Ostrom [OST 10] ajoute une troisième solution : la gestion par des communautés locales, leurs normes et arrangements institutionnels. Dans notre cas, le bien commun est l'espace proche et les acteurs sont des États, des entreprises, mais aussi des Universités. L'espace extra-atmosphérique est un bien commun typique; chaque État souverain a le droit de l'utiliser et aucun État ne peut empêcher les autres de l'utiliser. Les solutions proposées par Hardin (privatisation ou nationalisation) y sont difficilement applicables. Il Une forme de régulation par une autorité commune reconnue devrait alors fixer, pour le bien commun et les générations futures, une limite au droit individuel, comme pour les réglementations contre la pollution, stimulant alors l'innovation et l'émergence de nouveaux marchés.

\subsection{Ingénierie hétérogène : transformer les contextes}

La situation en matière de pollution spatiale n'est pas encore critique et les opérateurs de satellites disposent de deux options selon la taille des débris ${ }^{8}$. Pour atténuer les effets d'un impact sur leurs satellites, des blindages multi-couches peuvent être mis en place. Ils ne sont cependant efficaces que pour des débris ayant une taille inférieure au centimètre. Une meilleure conception du satellite peut aussi permettre de protéger les éléments fragiles en les plaçant sur des faces moins exposées. Pour faire face à des débris supérieurs à dix centimètres, des manœuvres d'évitement sont mises en place, mais elle ne convient que pour des débris catalogués et suivis par un système de surveillance. Ces méthodes accroissent toutefois le coût de la construction des satellites et affectent leurs caractéristiques (la masse notamment) et leurs performances. Par contre, il n'existe pas de solution pour les débris de taille

\footnotetext{
${ }^{7}$ https://avisdexperts.ch/videos/view/4209, dernière consultation le 16 avril 2019.

${ }^{8}$ http://debris-spatiaux.cnes.fr/, dernière consultation le 27 avril 2013.
} 
comprise entre un et dix centimètres. Le faible risque de collision ne pousse pas les acteurs à investir dans leur élimination active en ayant recours, par exemple, aux inventions de type Clean Space One. Les chercheur-euse·s du projet découvrent alors que leur belle invention se heurte à la dure réalité du peu d'incitations à œuvrer pour le bien commun. Leurs inventions risquent d'être condamnées à ne jamais devenir des innovations, à défaut d'être adoptées par des utilisateurs. Le suivi biographique de l'invention rend compte des incertitudes qui pèsent sur le projet et du fait que leur culture technique n'intégrait pas cette connaissance de l'inscription de la technologie en société.

La mise en place d'une régulation internationale semble devoir jouer un rôle primordial. Plusieurs agences nationales ont élaboré des règles de bonne conduite qu'elles s'imposent de respecter, ce qui induit un surcoût. L'adoption unilatérale de telles solutions comme Clean Space One induit, pour l'adopteur, un désavantage économique, même si elle accroit sa réputation en tant qu'acteur soucieux de l'environnement. Compétences technologiques, culture du domaine spatial et souci de la durabilité ne suffisent pas à placer sur orbite, dans la société, les belles inventions. Les constructeurs de satellite et leurs clients pouvant se passer des agences nationales qui établissent des règles de bonne conduite pour lancer leurs satellites, une régulation internationale semble s'imposer, mais se pose la question de l'entité régulatrice et contraignante qui pourrait le faire. L'ONU n'est pas une autorité régulatrice et n'est pas en mesure d'imposer une régulation internationale. Elle aurait plus de chance d'être respectée si elle faisait l'objet d'un consensus international. Un traité international signé par les différents acteurs pour réduire les déchets spatiaux serait la seule solution pour créer la société qui convient aux solutions technologiques proposées.

Ostrom [OST 10] analysant la perception des coûts et bénéfices des changements institutionnels montre que les acteurs accordent plus d'importance aux pertes qu'aux gains potentiels. Ils adoptent davantage les options permettant d'éviter de futurs inconvénients que celles visant au bien commun. Ils adoptent aussi davantage de nouvelles règles quand existent des indicateurs clairs de la dégradation d'une ressource. Or, dans le cas des débris spatiaux, la situation n'est pas clairement perçue par les acteurs. Etant plus attentifs aux coûts immédiats qu'aux bénéfices potentiels, les solutions (traités ou technologies) devraient éviter les coûts supplémentaires pour les futures missions spatiales.

L'enlèvement des débris n'est pas non plus une opportunité commerciale. Contrairement aux déchets terrestres, la masse et la dispersion des débris orbitaux rendent leur récupération et traitement peu apte à générer un profit économique. Même si l'idée de développer la capture et le transport des débris jusqu'à une station de recyclage, en orbite - avec tri et reconditionnement des matériaux, puis leur réutilisation grâce pour fabriquer d'autres satellites -, s'intègre progressivement dans la vision à long terme qui prévaut dans le domaine spatial ${ }^{9}$, ce recyclage n'est envisageable que pour les satellites actifs en fin dont la mission peut être prolongée ou modifiée. La participation de sociétés privées au nettoyage de l'espace dépendra alors du soutien financier des gouvernements ou, comme le pense Claude Nicollier, astronaute suisse et professeur à l'EPFL, consultant sur le projet Clean Space, de sociétés d'assurance.

Surgit alors un problème éthique. L'assainissement de l'espace proche de la Terre n'étant pas de la responsabilité d'un État ou d'une organisation, il suppose la coopération entre États et le déploiement d'une ingénierie encore plus hétérogène [LAW 89 ; HUB 14] composant avec les stratégies nationales. Clean Space One, satellite suisse, va récupérer Swiss Cube, mais s'il désorbite le satellite opérationnel appartenant à autrui engendrerait des conflits. Clean Space One ne peut pas se tromper de cible. Or, repérer SwissCube est un défi technologique car il est petit et de texture sombre. Si les gros débris sont bien localisés, les plus petits ne le sont qu'avec une incertitude d'environ cinq kilomètres. Les repérer, s'en approcher et les reconnaître sans se tromper implique de développer un système de propulsion, un système de capteur de rendez-vous - technologie qui n'existait pas - et un système de captation (en

${ }^{9}$ https://avisdexperts.ch/videos/view/7684, dernière consultation le 19 avril 2019. 
l'occurrrence un panier déployable de type packman. Or, SwissCube, vers 2020-2021, n'émettra plus aucun signal, ce qui rend sa détection difficile et exige le développement de senseurs non coopératifs.

La biographie de cette innovation intègre désormais des problématiques sociétales absentes de la culture technique des ingénieur.e.s impliqué.e.s. Devant agir dans un monde où d'autres acteurs doivent contribuer à la résolution des problématiques environnementales, éthiques, économiques et politiques, les seules performances techniques et la prise en compte des contraintes du spatial ne suffisent pas à assurer le succès. L'innovation dépend aussi de la transformation des règles du jeu, politiques, éthiques et économiques, à moins que Clean Space One ne conçoive une solution qui ne dépende pas de tels changements. Le but du projet n'était pas seulement de mettre des élèves ingénieur.e.s en situation « réelle » et d'accroître la réputation académique de leur institution, mais d'innover et de contribuer à un développement durable de l'espace. Le projet est pensé comme une étape de démontration de la validité technologique de solutions à reprendre par d'autres projets, notamment Clean Space Two qui serait conçu et optimisé pour aller chercher plusieurs débris et réduire le coûteux lancement dans l'espace. Leur diffusion dépend de la démonstration technique et de la commercialisation de la solution à un coût intéressant pour les clients potentiels. Le futur du projet prendrait la forme d'une entreprise qui propose une solution de désorbitage et éviterait aux autres acteurs de solutioner eux-mêmes la fin de vie de leur satellite, tout en se montrant «environmentally friendly ». Cela implique de proposer un système de désorbitage compétitif par rapport à une solution intégrée dans la mission. Si la démonstration technique et économique était faite et moyennant une régulation incitative, la solution serait reprise et «se » diffuserait au sein de la communauté spatiale.

\subsection{Une culture technique qui intègre la réalité du monde}

En 2013, le Swiss Space Center annonce la création d'un partenariat avec l'entreprise Swiss Space Systems $(\mathrm{S} 3)^{10}$ qui développe un système de lancement indépendant et « écologique » et pourrait lancer Clean Space One en le plaçant dans un petit véhicule spatial réutilisable, adossé à un Airbus A300 certifié pour le vol zéro-G. Une fois arrivée à la bonne altitude, la navette allumerait ses moteurs-fusées pour monter en orbite où elle lâcherait à le satellite. La mission terminée, la navette revient sur terre jusqu'à son aérodrome de départ. Le partenariat avec S3 serait un bout de solution pour le financement de Clean Space One et pour assurer un lancement de précision (la précision du rendez-vous avec Swiss Cube est essentielle) ; inversément, il contribuerait à la réputation «écologique » de S3 et prouverait que des missions exigeantes peuvent être réalisées avec ce nouveau lanceur. S3 assurerait alors l'industrialisation de CleanSpace et sa diffusion auprès de clients potentiels. Ce partenariat conforte le réseau des acteurs associés à CleanSpace One, mais n'est qu'une première étape vers l'enrôlement d'autres acteurs, adopteurs potentiels, condition sine qua non de succès en tant qu'innovation. Les porteurs de l'invention étoffent leur culture de l'innovation en intégrant le souci de la collaboration, du réseau, de la finance et des conventions internationales à leur culture technique, à la compréhension de la complexité des problèmes et des contraintes du milieu, ainsi qu'aux capacités à retraduire dans le travail technologique, la connaissance des contextes d'usage. S3, malheureusement, est mise en faillite en 2016 et disparait en 2017, mais en avril 2019, le projet CleanSpace One crée une startup, renforçant encore cette culture de l'innovation. ${ }^{11}$

En 2018, un satellite concurrent RemoveDebris, porté par un réseau européen d'entreprises (Airbus, Surrey Satellite Technology Ltd) et d'institutions de recherche spatiales (INRIA, Université de Surrey, de Stellenbosch et de Neuchatel) est lancé et teste avec succès la capture d'un « faux » débris au moyen d'un filet, une technique d'identification de débris, en février 2019, et une technique de harponnage en mars 2019. ${ }^{12}$ Plusieurs démonstrateurs sont aussi en cours de développement dans le monde. Les premières missions opérationnelles américaines datent de 2018. La biographie de l'innovation devient alors celle d'une coopération-compétition entre assemblages d'acteurs

\footnotetext{
${ }^{10}$ https://fr.wikipedia.org/wiki/Swiss Space Systems, dernière consultation le 12 mai 2019.

11 https://actu.epfl.ch/news/how-a-startup-plans-to-clean-up-space-2/, dernière consultation le 12 mai 2019.

12 https://www.csem.ch/Page.aspx?pid=53155, dernière consultation le 19 avril 2019. 
hétérogènes et de technologies. Elle suppose de comprendre les dynamiques technologiques dans ce segment de la société. Elle articule des défis scientifiques et techniques à des problématiques d'incitation et de régulation. Elle contribue à créer des technologies et les consortium qui les portent, mais aussi des savoirs, des organisations, des visions partagées et des métiers d'éboueur de l'espace [BON 16], et de développer la culture technique du domaine spatial, déjà imprégné de cultures scientifiques, politiques et industrielles.

\section{Conclusion : le façonnage de l'innovation par la tragédie des communs}

Dans cet article, nous avons rendu compte de la biographie d'une invention qui cherche à devenir innovation. Nous avons vu qu'elle est le fruit du travail de divers acteurs (académiques, industriels, agences spaciales nationales et internationales, instances de régulation, étudiant $\cdot \mathrm{e} \cdot \mathrm{s}$, etc.) qui œuvrent dans un contexte fait de concurrence économique internationale, de prise de conscience vis-à-vis des problématiques environnementales, de contribution au bien commun et au bien-être de l'humanité, de souci du respect du secret industriel ou militaire des nations, etc., ainsi que d'autres inventions (composants commercialement disponibles, technologies et standards mis à disposition, etc.). Le déclencheur ayant permis l'impulsion du programme Clean- $m E$ est le retrait du soutien de la part d'une entité internationale à une équipe de chercheur-euse-s, enseignant.e.s et étudiant.e.s. Cette dernière, voulant prouver sa valeur au reste du monde, a souhaité poursuivre l'aventure malgré le fait de ne pas avoir intégré la préoccupation émergente pour la propreté de l'espace. De là est né un autre projet visant à démonstrer sa faisabilité technique, mais qui se heurte à l'adoption de la solution par d'autres acteurs de l'espace.

Une invention devient innovation si elle est adoptée par des utilisateurs qui y trouvent un intérêt, économique par exemple. Or, tel n'est pas le cas des inventions qui servent avant tout le bien commun. Or, la réglementation internationale de 1995, mondialement agréée en 2002, afin d'éviter de générer des débris spatiaux, n'est suivie qu'à $50-60 \%{ }^{13}$; les opérateurs ne sont guère incités à sauver le bien commun, en cessant son exploitation ou en investissant pour son nettoyage. Le sort de l'invention étudiée ici dépend alors d'une validité démontrée des solutions technologiques mais aussi de la mise en place d'un environnement favorable : réglementation, autorité reconnue qui veille à la sauvegarde de l'intégrité de la ressource et création d'un nouveau métier. L'innovation dépend ainsi de dynamiques politiques et des sensibilités éthiques et environnementales. Sa biographie complète la culture technique des ingénieur.e.s impliqué.e.s. Avec la tragédie des communs, elle souligne l'importance de valeurs, savoirs et pratiques en jeu si se réalise l'exploitation de la Lune, de Mars et d'autres astéroïdes.

Cette problématique de la pollution spatiale prépare l'émergence de nouveaux métiers, marchés et mécanismes de régulation qui conditionnent le déploiement d'innovations. Pour que la culture technique permette à un collectif d'agir avec succès dans le monde en intègrant ces éléments, les biographies d'innovations l'enrichiraient en évitant de réduire l'invention à la recherche d'une solution à un problème, l'innovation comme sa mise en œuvre et l'explication du succès par le génie d'individus ou les caractéristiques de la technique. La biographie d'innovations en cours aide à comprendre les processus sociotechniques de leur genèse et apporte des connaissances décisives aux concepteurs.

\section{Références}

[AKR 91] AKRICH M., CALLON M., LATOUR B., « À quoi tient le succès des innovations », in ViNCK D. (éd.), La gestion de la recherche. Nouveaux problèmes, nouveaux outils, De Boeck, Bruxelles, p. 25-76, 1991.

[BON 16] Bonnal C., Pollution spatiale : l'état d'urgence, éd. Belin, Paris, 2016.

\footnotetext{
${ }^{13}$ https://avisdexperts.ch/videos/view/6863, dernière consultation le 16 avril 2019.
} 
[CHA 09] Chalas Y., GILbERT C., VINCK D. (dir.), Comment les acteurs s'arrangent avec l'incertitude ?, Edition des Archives Contemporaines, Paris, 2009.

[CHO 17a] Chouteau M., Forest J., NGuyen C., «Culture technique, Culture d'innovation : présentation générale », Technologies et innovation, $\mathrm{n}^{\circ} 2,2017$.

[CHO 17b] ChOuteau M., Forest J., NGuYen C., "Quand la culture d'innovation fait écran à la culture technique », Technologies et innovation, $\mathrm{n}^{\circ 2}$, 2017.

[DID 17] DIDIER J., «Culture technique et culture de l'innovation: réconcilier ce tandem par le biais de la conception », Technologies et innovation, $\mathrm{n}^{\circ} 2,2017$.

[EDG 98] Edgerton D., «De l'innovation aux usages. Dix thèses éclectiques sur l'histoire des techniques », Annales. Histoire, sciences sociales, ${ }^{\circ} 4-5$, p. 815-837, 1998.

[ESA 06] ESA. Position Paper on Space debris mitigation: Implementing zero debris creation zone, ESA SP-1301, 2004, Noordwijk, The Netherlands, 62 p., 2006, http://www.esa.int/esapub/sp/sp1301/sp1301.pdf (dernière consultation le 1.11.18)

[ESA 18] ESA. Space debris by the numbers, 2018.

https://www.esa.int/Our Activities/Operations/Space Debris/Space debris by the numbers (dernière consultation le 29.10.18)

[GOD 17] GODIN B., VINCK D. (eds.), "Critical studies of innovation: Alternative approaches to the pro-innovation bias", Edward Elgar, Cheltenham, 2017.

[GRA 13] Gravrilovich I., Shea H., Richard M., Clean Space One Gripper Report, Phase 0, Lausanne : LMTS, Swiss Space Center, 57 p., 2013, http://infoscience.epfl.ch/record/188245/files/DEMES\%20GRIPPER\%20report\%20\%20Irina.pdf (dernière consultation le 1.11.18).

[HAL 14] Hall L., The History of Space Debris, Space Traffic Management Conference, 2014, https://commons.erau.edu/stm/2014/thursday/19 (dernière consultation le 1.1q.18).

[HAR 68] HARDIN G., "The Tragedy of the Commons", Science, vol. 162, n 3859, p. 1243-1248, 1968.

[HUB 14] HUBERT M., VINCK D., « Des pratiques d'ingénierie aux transitions sociotechniques. Retour sur la notion d'ingénierie hétérogène dans le cas des micro- et nanotechnologies », Revue d'anthropologie des connaissances, vol. 8, n², p. 361-389, 2014.

[JAC 17] JACOMY B., «La culture technique et le monde contemporain », Technologies et innovation, $\mathrm{n}^{\circ} 2,2017$, DOI : 10.21494/ISTE.OP.2017.0159.

[LAT 88] LATOUR B. (as "Jim Johnson"), "Mixing Humans and Nonhumans Together: The Sociology of a Door-Closer," Social Problems, Special Issue: The Sociology of Science and Technology, n 35, p. 298-310, 1988.

[LAT 91] Latour B., "Technology is Society Made Durable", in LAW J. (ed.), A Sociology of Monsters: Essays on Power, Technology, and Domination, n³8, New York: Routledge, p. 103-132, 1991.

[LAT 05] LATOUR B., Reassembling the social, Oxford University Press, Oxford, 2005.

[LAW 89] LAW J., "Technology and heterogeneous engineering: the case of Portuguese expansion", In: BIJKER W., Hughes T., PINCH T. (eds.) The social construction of technological systems. New directions in the sociology and history of technology. MIT Press, Massachusetts, p. 111-134, 1989.

[MAN 08] MANACH J.-M., "Quand "les" nanotechnologies ont détourné "la" nanotechnologie de son projet durable », Internetactu.net, 03/09/2008, http://www.internetactu.net/2008/09/03/quand-les-nanotechnologies-ont-detourne-lananotechnologie-de-son-projet-durable/

[NOC 09] Noca M., Jordan F., Steiner N., Choueri T., ScheidegGer N., Peter-Contesse H., Krpoun R., Shea H., GEORGE F., BorgeAUd M., Lessons learned from the First Swiss Pico-satellite: SwissCube., $23^{\text {rd }}$ Annual AIAA/USU, Conference on Small Satellite, Logan (USA), August 2009, $20 \quad$ p. http://digitalcommons.usu.edu/cgi/viewcontent.cgi?article=1345\&context=smallsat (dernière consultation le 1.11.18).

[OST 10] OSTROM E., BAECHLER L., Gouvernance des biens communs - Pour une nouvelle approche des ressources naturelles, De Boeck, Bruxelles, 2010.

[RIC 13] Richard M., Kronig L., Belloni F., Rossi S., Gass V., Paccolat C., Thiran J.P., Aroami S., GaVRILOVICH I., SHEA H., Uncooperative Rendezvous and Docking for MicroSats : The case for CleanSpace One, $6^{\text {th }}$ International Conference on Recent Advance in Space Technologies, RAST 2013, Istanbul (Turkey), 11 p., http://space.epfl.ch/files/content/sites/space/files/CleanSpaceOne\%20RAST\%20paper,\%20June\%202013.pdf (dernière consultation le 1.11.18). 
[SWI 10] SWISS SPACE CENTER. Clean-mE: an orbital debris removal mission, Phase 0, SSE Fall 09 report, EPFL, Lausanne, 2010.

[VIN 14] VINCK D., « Pratiques d'ingénierie. Les savoirs de l'action », Revue d'anthropologie des connaissances, vol. 8, $\mathrm{n}^{\circ} 2$, p. 225-243, 2014.

[VIN 18] Vinck D., CAMUS A., JATON F., OBERHAUSER P.-N., «Localités distribuées, globalités localisées : actions, actants et médiations au service de l'ethnographie du numérique », Symposium, vol. 22, n¹, p. 41-60, 2018.

[WIL 14] WiLliams B., FigueIREDo J., «L'ingénierie hétérogène portugaise. À la découverte de solutions viables, Revue d'anthropologie des connaissances, vol. 8, n² 2, p. 279-306, 2014.

[WAN 13] WANG P., Tragedy of Commons in Outer Space - The casa of Space Debris, 64th International Astronautical Congress 2013, 56th IISL Colloquium on the Law of Outer Space, Beijing, China, 19 p., http://ssrn.com/abstract=2260856 (dernière consultation le 1.11.18). 